\title{
Presence of a Juvenile Idiopathic Arthritis and Chronic Granulomatous Disease in a Child
}

\author{
Taravat Sadrosadat ${ }^{1}$; Vahid Ziaee ${ }^{2,3,}$; Yahya Aghighi ${ }^{3,4}$; Mohammad Hassan Moradinejad ${ }^{1,3}$; \\ Masoud Movahedi ${ }^{2}$ \\ ${ }^{1}$ Children's Medical Center, Pediatrics Center of Excellence, Tehran University of Medical Sciences, Tehran, IR Iran \\ ${ }_{2}^{2}$ Pediatric Rheumatology Research Group, Rheumatology Research Center, Tehran University of Medical Sciences, Tehran, IR Iran \\ ${ }^{3}$ Department of Pediatrics, Tehran University of Medical Sciences, Tehran, IR Iran \\ 4 Valiesar Hospital, Imam Khomeini Complex Hospital, Tehran University of Medical Sciences, Tehran, IR Iran \\ *Corresponding author: Vahid Ziaee, Pediatric Rheumatology Research Group, Rheumatology Research Center, Tehran University of Medical Sciences, Tehran, IR Iran. \\ E-mail: ziaee@tums.ac.ir
}

Received: March 3, 2015; Revised: March 14, 2015; Accepted: March 26, 2015

Keywords: Arthritis Juvenile; Granulomatous Disease Chronic; infection

\section{Dear Editor,}

Chronic Granulomatous Disease (CGD) is a result of deficient oxidative burst and impaired generation of reactive oxygen species due to mutations in genes encoding for NADPH oxidase complex (1). CGD usually presents with recurrent infections such as pneumonia, lymphadenitis osteomyelitis and hepatic or other abscesses (2) Rheumatologic disorders is a rare manifestation of first presentation of CGD.

Juvenile idiopathic arthritis (JIA) is the most common chronic rheumatic disease among children. It collectively refers to a group of chronic arthritis of at least six weeks duration in children or adolescents under the age of 16 (3). JIA is a common problem in immuodefficiency diseases but is not common in CGD. However, there is a little reports of concordances of rheumatologic diseases and immunodeficiencies. In addition to increased susceptibility to infections in patients with CGD, a higher incidence of sterile inflammatory disorders in these patients has been noted (4).

Our patient is a young boy who was born to the related parents whose their previous two children were dead because of myelomeningocele and anencephaly, respectively. The patient was 2 years old when he suffered from pelvic pain .Considering clinical findings in addition to $4+$ RF, anti-CCP: 200 and negative HLA B5, B8 and B27, he was diagnosed as poly articular JIA. He was on medication (prednisolone and metotraxate) for years without remission but he had any complications.

When he was 6 years old he experienced several episodes of sinusitis, cervical lymphadenitis and pneumonia. His lymphadenitis was managed surgically because it did not respond to non invasive therapies. In his ad- mission to hospital because of pneumonia several work ups were performed as a result of unsuccessful antibiotic therapy. Bronchoscopy revealed nothing. Complete bold count was normal $(\mathrm{WBC}=9300$ with $63 \%$ PMN $)$. CD markers and all serum immunoglobulins level were in normal range. Nitroblue Tetrazolium (NBT) test in patients was 0 but in father and mother's patient was 87 and 98, respectively (normal range: 52 - $88 \mu \mathrm{g}$ ). In addition, activity dihydrorhodamine (DHR) Flow Cytometric test reduced in patient $(\mathrm{DHR}=8)$ and in father and mother's patient was 27.5. The patient underwent CGD treatment based on the laboratory data, and clinical improvement was soon appeared. Now he is on JIA and CGD treatment with poor control of his joint involvements and he gradually developed ulnar and plantar deviation which has made him using braces. There are some reports of concordances of rheumatologic diseases and immunodeficiencies. There are reports of CGD accompanying Kawasaki disease (5) DiGeorge and JIA (6), CGD in a girl with SLE and CGD in a patient with antiphospholipid antibody syndrome (7). Lee and Yap were among the first researchers who reported a patient with CGD and JIA. Despite our patient, they reported a girl who developed JIA after CGD (8). De Ravin and colleagues also reported different cases of CGD which gradually developed different rheumatologic disorders among them there is a patient with JIA who had been previously diagnosed as CGD (7). The difference between these reports and ours was the priority of immunodeficiency in their cases and rheumatologic disorder in our case.

Some authority believe CGD might itself be an associated risk factor for developing immune-based inflam-

Copyright (C) 2015, Growth \& Development Research Center. This is an open-access article distributed under the terms of the Creative Commons Attribution-NonCommercial 4.0 International License (http://creativecommons.org/licenses/by-nc/4.0/) which permits copy and redistribute the material just in noncommercial usages, provided the original work is properly cited. 
matory disorders (4). However, there are authors that believe JIA acts as a predisposing factor for autoimmunity (7). Collectively, we believe that immunodeficiency and rheumatologic disorders are the two ends of a spectrum in which a patient might demonstrate different manifestations of each. So, for each immunodeficient or rheumatologic patient every sign and symptoms should be precisely considered because it might be a presentation of a new disorder.

\section{References}

1. Induction of regulatory $\mathrm{T}$ cells by macrophages is dependent on production of reactive oxygen species. Kraaij MD, Savage ND, van der Kooij SW, Koekkoek K, Wang J, van den Berg JM, et al. Proc Natl Acad Sci U S A. 2010;107(41):17686.

2. Kliegman R, Stanton B, Schor N, Geme J, Behrman R. Nelson Text
Book of Pediatrics. 19th ed. Philadelphia: Elsevier; 2011. pp. 745-6.

3. Juvenile idiopathic arthritis-associated uveitis: a nationwide population-based study in Taiwan. Yu HH, Chen PC, Wang LC, Lee JH, Lin YT, Yang YH, et al. PLoS One. 2013;8(8):e70625

4. Sarcoidosis in chronic granulomatous disease. De Ravin SS, Naumann N, Robinson MR, Barron KS, Kleiner DE, Ulrick J, et al. Pediatrics. 2006;117(3):e590.

5. Chronic granulomatous disease associated with atypical Kawasaki disease. Yamazaki-Nakashimada MA, Ramirez-Vargas N, De Rubens-Figueroa J. Pediatr Cardiol. 2008;29(1):169.

6. Association of juvenile idiopathic arthritis and digeorge syndrome; a case report. Salehzadeh F, Bagheri A. Iran J Pediatr. 2014;24(3):334

7. Chronic granulomatous disease as a risk factor for autoimmune disease. De Ravin SS, Naumann N, Cowen EW, Friend J, Hilligoss D, Marquesen M, et al. J Allergy Clin Immunol. 2008;122(6):1097.

8. Polyarthritis resembling juvenile rheumatoid arthritis in a girl with chronic granulomatous disease. Lee BW, Yap HK. Arthritis Rheum. 1994;37(5):773. 